\title{
Measurement and Evaluation of the Bioelectrical Impedance of a Rubber Humanoid Phantom Wrapped with Gel Sheets
}

\author{
Shinji Kawakura*, Yoshihiro Nakabo, and Kiyoshi Fujiwara \\ National Institute of Advanced Industrial Science and Technology (AIST), Department of Information \\ Technology and Human Factors, Robot Innovation Research Center 1-1-1 Umezono, Tsukuba, Ibaraki \\ 305-8560 Japan. \\ * Corresponding author. Tel.: +81-80-9781-1535; email: s.kawakura@aist.go.jp \\ Manuscript submitted August 30, 2017; accepted November 5, 2017. \\ doi: 10.17706/ijbbb.2018.8.2.155-163
}

\begin{abstract}
This study aimed to estimate the impedance values of an originally made, artificial rubber phantom attached with one elastic gel sheet using Physion MD (Doctor of Medicine). The electromagnetic characteristics of the material were similar to human body. In previous studies, specially designed bioelectrical impedance acquisition systems and MRI (magnetic resonance imaging) methods were used to independently and repetitively measure bioelectrical impedance and serial cross-sectional images of the trunk, forearms, upper arms, lower legs, and thighs of healthy adult men. Bioelectrical impedance was significantly correlated to muscle and fat volumes measured by MRI. Based on this relationship, the estimated values of muscle and fat could also be calculated from the bioelectrical impedance. However, past methodologies have various limitations. In this current, subsequent research, only impedance values were measured, while varying superficial variables (e.g., attaching gel sheets, changing electrode types, and changing the insertion depth of needle-shaped electrodes) This present study indicates that bioelectrical impedance analysis for this rubber phantom including the version with specific gel sheets attached and the version containing normal saline solution is a promising physical simulator, and may be useful for the prediction of not only virtual muscle volume, however, also diverse inner body conditions.
\end{abstract}

Key words: Artificial rubber phantom, gel sheet, normal saline, Physion MD, bioelectrical impedance.

\section{Introduction}

In recent years, various kinds of electric stimulations, human body measurements, and social science-based cares have been growing in the fields of medicine, massage therapy, simple symptomatic therapies, and sports science [1]-[4]. Both outdoor measurement systems and indoor electric embedded equipment for various real-time body measurements have been evolving each year; for instance, pacemaker data and pulse rate meters for common body conditions [4]-[6]. For a long time, researchers, healthcare personnel, and engineers who used electrical-based systems could obtain skin and internal body impedance values that required them to determine a baseline for current sources and voltage-measuring circuits. Various system designers who use two or four electrode instruments should be particularly careful, because measurements with real and unreal bodies, which do not contain skin or skin-simulating substances, may not yield similar results to measurements that do include skin or skin-simulating substances. On the other hand, bioelectrical engineering in the medical field and in general has been progressing; scientists are 
developing analysis methods and systems that target both the surface of the human body and the inner organs and muscles, to take measurements and to act as a countermeasure against sudden electric accidents and the like [7]-[16]. However, there was some variation in the assumed (targeted) subjects' race, age, career, and other parameters. For such experiments in these scientific fields, researchers often utilize 1) computer program-based simulators, 2) non-contact methods, and 3) real human bodies, as long as these experimental settings are safe enough in light of official regulations and public laws [17]-[19]. However, in these scientific areas, the targets of these measurements were limited to living human bodies, heads, and limbs, and have not included non-living robots and dolls until now. There are rigid regulations and difficulties concerning the safety, research ethics, and legal aspects of these studies. That is why researchers and medical service workers cannot cut, pierce, and embed electronic modules (pace maker, electrical stimulation device, etc.) in human bodies for experimental purposes only. To avoid such risks in this study (e.g., cutting a real body, inserting electrodes, and changing superficial skin conditions), the authors selected and measured one artificial custom-made phantom as a physical simulator. The creators carefully designed the phantom, beginning from the early phase of production of the metallic mold. The value of saline solution ( $0.18 \mathrm{wt}$ (weight) \%) and the phantom's rubber are very similar at a measurement frequency of $3000 \mathrm{MHz}$ concerning the real part of conductance rate $\kappa$; hence, the selection of this phantom as a physical experimental simulator is appropriate for the goals of this study. The manufacturer of this phantom, Asahi Rubber Inc., Japan and researchers (e.g., Takashi Hikage, Graduate school of Hokkaido University, Sapporo, Japan), have been developing and producing such phantoms for years, with the purpose of collecting data relating to electromagnetic characteristics. The phantom was originally to be used for diverse experiments regarding the propagation and diffraction of radio waves from common cell phones. It has specific electromagnetic characteristics that are relatively close to that of a real human body. The conductance rate $\kappa[\mathrm{S} / \mathrm{m}]$ of the specific rubber of the phantom, a significant electromagnetic characteristic. In the first phase of this study, the authors used the Physion MD (Doctor of Medicine) to measure the phantom's default body according to the official instructions. In the second phase, the authors partially wrapped the surface of the phantom with two types of artificial gel sheets ("NR" type and "SRA" type, manufactured by Sekisui Kasei Manufacturing Inc., Japan). In this study, the results of NR are shown. All eight gel sheets used were cut to $50 \mathrm{~mm} \times 50 \mathrm{~mm}$ squares by the authors, and attached on the Physion MD's default measurement point. Furthermore, in the third phase of this study, the authors used brush and a spray bottle to fill the empty spaces between the phantom and the gel sheets, as well as the subtle cracks of the phantom, with normal saline solution (NSS, $0.9 \mathrm{wt} \%$ ). By focusing on both recent and traditional bioelectrical techniques, the authors verified the usefulness of this experiment to some extent. Thereafter, the present study constructed and demonstrated the simulation and measurement system for use in an indoor experimental laboratory, and other testing sites. Thus, the purpose of this present research is to prove the utility of this phantom in the fields of medication, massage and symptomatic therapy, and sports science.

\section{Theory and Methods}

\subsection{Theory}

The difficulty of measuring impedance of various body parts has been argued for many decades [11], [18], [20]-[24]. In previous studies, researchers based impedance indexes on $L^{2} / Z$ ( $L$ : body segment length; $Z$ : impedance) for every body-segment, i.e., the ratio of the squared segment length to the impedance. In this current study, the authors only measure impedance values, while changing superficial variables (e.g., by attaching specific gel sheets, changing electrode types, and changing the insertion depth of needle-shaped electrodes). In this study, the authors report in detail the numerical differences of the impedance 
parameters, as well as the mathematical relationship between past data from real human bodies and the data from this phantom. The authors show that the series of impedance values produced from the phantom in the three different aforementioned conditions (the default phantom, with gel sheets attached, and with NSS in addition to gel sheets attached) closely modeled a real human body wrapped with elastic skin. The authors selected this phantom for its physical specifications: appearance, conductance rate $[\mathrm{S} / \mathrm{m}]$, and NSS-containing gel sheets; as well as its stature. At $165.0 \mathrm{~cm}$, the phantom is almost same stature as an average middle-aged to elderly (60-69 years old) Japanese man, who stand, on average, at $165.9 \mathrm{~cm}$ (Ministry of Health, Labor and Welfare, Japan, 2013 [25]). Researchers, healthcare personnel, and bio-engineers are required to achieve relatively high input impedances over the measurement frequency range, in order to prevent the imbalances in skin impedance from converting common mode voltages to differential voltages. Additionally, the sizes of body parts are assumed to be the same. Furthermore, the angle of both infra-axillary sites and crotches were set at 30 degrees. In order to use these impedance systems in medical institutions or medical research institutions in basic studies, the developers have intentionally adjusted to these assumptions.

\subsection{Methods}

In the first phase of this study, the authors measured the phantom's default body according to the official instructions. In the second phase, the authors partially wrapped the phantom's surface with two types of artificial gel sheets (NR and SRA, manufactured by Sekisui Kasei Manufacturing Inc., Japan), and showed data for the results of NR. The authors cut all eight gel sheets to $50 \mathrm{~mm} \times 50 \mathrm{~mm}$ squares, and attached them to the default measurement points on the Physion MD (Fig.1 and 2).

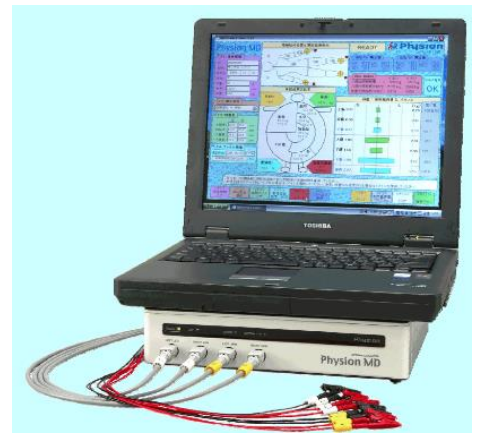

Fig. 1. Physion MD.

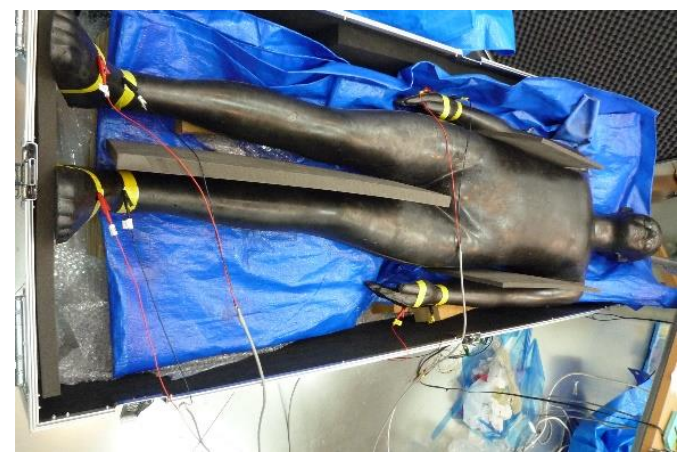

Fig. 2. Rubber humanoid phantom connected to Physion MD.

In the third phase, the authors used a common brush and spray bottle to fill the empty spaces between the gel sheets and the phantom, and subtle cracks of the phantom with NSS ( $0.9 \mathrm{wt} \%)$. The relative direct current (DC) resistance of the NR gel sheet is $2 \times 10^{4} \Omega / \mathrm{cm}$, with an impedance of $100 \Omega / \mathrm{cm}^{2}$, and these 
values change according to measurement frequencies. The thickness of the NR gel sheet is around $1.0 \mathrm{~mm}$. There is various antecedent research concerning the electric resistance and impedance of human skin only [6], [10], [11]. Generally, the DC resistance of dry human skin is around $1 \times 10^{4} \Omega / \mathrm{cm}$ (around $800 \Omega / \mathrm{cm}$ in wet conditions). The thickness of the epidermis is around 0.06 to $0.2 \mathrm{~mm}$, while the dermis is 2.0 to $2.2 \mathrm{~mm}$ thick. Considering this, the authors selected the most adequate gel sheet that could be bought. Regarding specific aspects of electronic engineering, other researchers have noted that skin impedance at low-frequency bands occasionally reaches extremely high values. Variations in the series of impedance values are difficult to incorporate into the reconstruction algorithm, and should produce large distortions [26]. On the other hand, the impedance of muscles and organs could also reach rather high levels. In general, subjects' skin, muscles, organs, bones, and flowing blood have diverse characteristics in reality. That is why simulating a human body is extremely complicated. However, a downside of measuring non-living objects is that researchers have difficulties discussing the electronic complexity. In this study, the authors calculated the precision of this using phantom quantitatively using standard bioelectric measurements in the field of sports science, and then certified and demonstrated its validity and reliability. Considering the aforementioned complexity of electronic specifications and features, the authors have conducted this study with only one phantom and measurement device. The authors selected Physion MD (licensed by Nippon Shooter Inc., Japan) as the equipment used to measure impedance [18], [23], [24], [27]-[29]. The specifications of the Physion MD were as follows: the measurement frequency was $50 \mathrm{kHz}$, the measurement current was $500 \mu \mathrm{Arms}$, and the measurement range was 10-1500 $\Omega$. The accuracy was $\pm 1 \%$ $\pm 0.5 \Omega$ at an impedance of $50-1000 \Omega$, or $\pm 2 \% \pm 0.5 \Omega$ at other impedance ranges, and the resolution was $0.1 \Omega$. The measurements were made based on Bio Impedance Analysis (BIA), which has been evolving until now. Measurements were made at common frequencies in this field; designers and medical scientists of electrical impedance tomography typically use $50 \mathrm{kHz}$. The theory behind the BIA method, as annotated by inventors of all the Physion series (Dr. Masuo et al.), states that the impedance $(Z)$ of a column-shaped conductor is defined from the volume resistivity $(\rho)$ and the cross-section area $(A)$. However, for measurements made at high frequencies, the $Z$ of a column-shaped conductor is proportional to the length of $L$, however is inversely proportional to the cross-section $A$. The volume of a conductor is proportional to $L^{2}$, however is inversely proportional to $Z$. The value of $L^{2} / Z$ is referred to as the BI index, which is proportional to the volume of conductors [1], see equations (1), (2) and (3)).

$$
\begin{gathered}
Z=\rho \times(L / A)(\Leftrightarrow A=(\rho \times L) / Z) \\
V=A \times L \\
V=\{(\rho \times L) / Z\} \times L=\rho \times\left(L^{2} / Z\right)
\end{gathered}
$$

Bioelectrical impedance is typically measured using a single frequency and the inductive electrode method. In previous foremost examples of such measurements, researchers have used the Physion MD [23], [24], [28] and Red Dot 2330 (3M Health Care / 3M United States, MA), a type of common gel pad, for each electrode. The authors selected electrocardiography electrodes for the placement of $2.2 \mathrm{~cm} \times 2.2 \mathrm{~cm}$ of Red Dot 2330 patches, which have a thickness of about $0.5 \mathrm{~mm}$. Previous studies have proven the validity of Physion MD and its peripheral systems. In addition, the authors selected one set of needle-shaped stainless electrodes (2 mm diameter, $120 \mathrm{~mm}$ length; Kyozai club $\mathrm{T} \& \mathrm{Y}$ Inc., Japan) and inserted eight such electrodes on the measurement points of Physion MD in the direction of the center of each of the phantom's limbs. Thereafter, the authors measured the effects of changing the insertion depth of the stainless 
electrode, from 1.0 to $4.0 \mathrm{~cm}$ in depth, with $1.0 \mathrm{~cm}$ increments. The thickness of the phantom's wrists, ankles, and tips of both hands and feet were around 1.5 to $4.5 \mathrm{~cm}$. As such, considering the directed measurement points of Physion MD, the authors thought that the candidate depths $(1.0$ to $4.0 \mathrm{~cm})$ were adequate for measurement. The body parts measured in the phantom were 1) between both arms, 2) the right arm, 3) the left arm, 4) between both legs, 5) the right leg, 6) the left leg, 7) between the right arm and leg, 8) part 1 of the body trunk, 9) between the left arm and leg, 10) part 2 of the body trunk, 11) between the right arm and left leg, 12) part 3 of the body trunk, 13) between the left arm and right leg, and 14) part 4 of the body trunk. Parts 1, 2, 3, and 4 of the body trunk overlapped due to the different current routes used for measurement. The inventors of the Physion series, including the MD, and the researchers in previous similar studies proposed these partitions [23], [24], [28].

\section{Results}

The authors calculated and showed the average impedance values and standard deviations (S.D.) in ohms of each body part measured at $50 \mathrm{kHz}$ (Fig. 3-5). In Fig. 3, the authors contrasted the impedance data from the default phantom with data from a previous similar study, the "Applicability of a segmental bioelectrical impedance analysis for predicting the whole body skeletal muscle volume", written by Tanaka et al. (2007) [28]. Fig. 3 shows that the numerical data from this study were below $50 \%$ of the data shown by Tanaka et al. (2007) [28]. The authors speculate that this was due to big or small internal cracks, and differences in composition (mainly rubber, silicon and NSS). In addition, phantom developers and the authors should reselect the compositions around this measurement frequency.

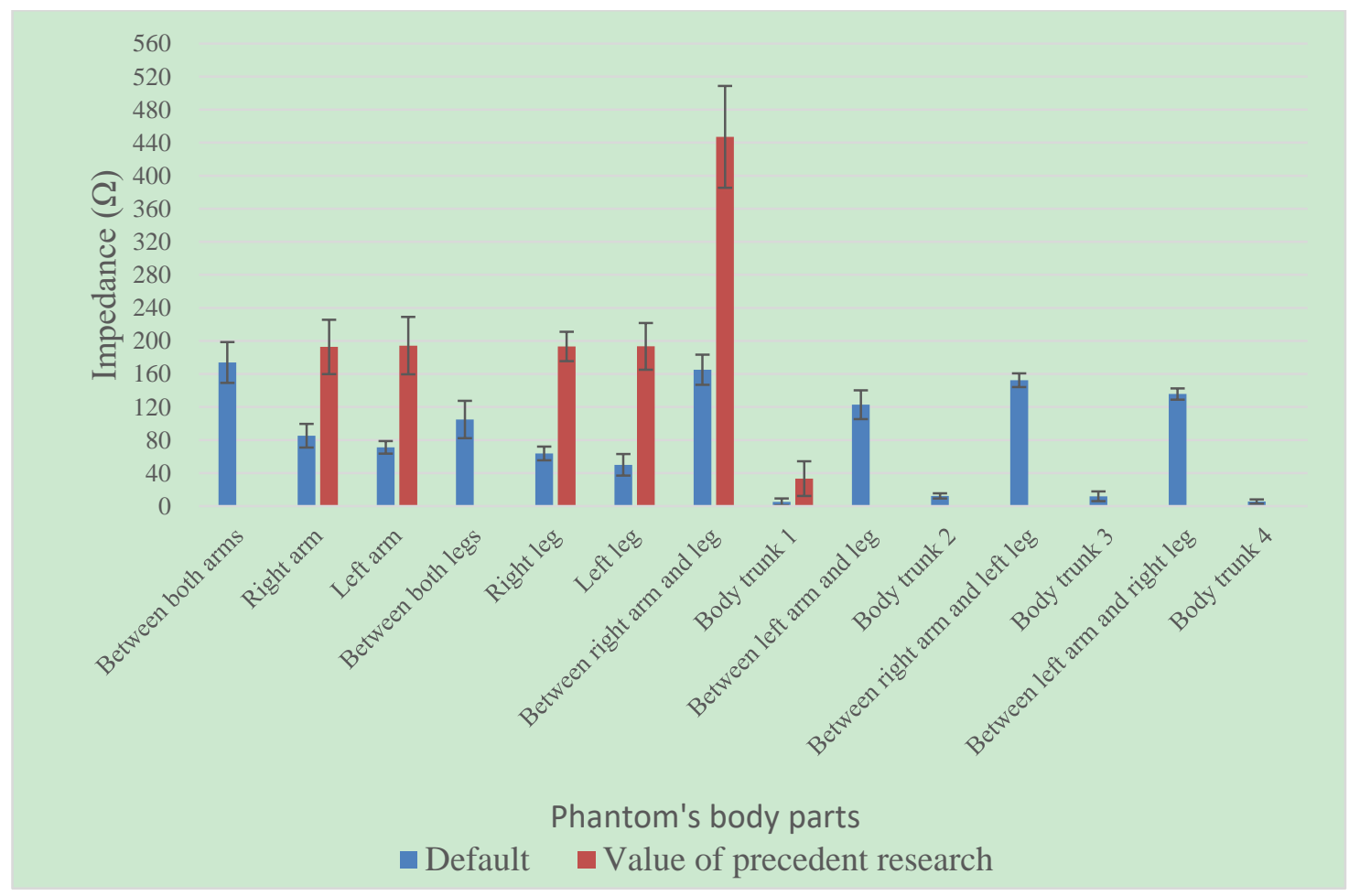

Fig. 3. A comparison between data from this study and a previous similar study (Tanaka et al. (2007)).

In Fig. 4, the authors calculated and showed the average impedance values and S.D. in ohms of each body part at $50 \mathrm{kHz}$, at insertion depths of stainless needle-shaped probes from 1.0 to $4.0 \mathrm{~cm}$. In Fig. 5, the authors show the average impedance values and S.D. in ohms of each body part in three conditions: the default phantom (unwrapped), the phantom with NR gel sheets attached, and the phantom with NSS in 
addition to the attached NR gel sheets.

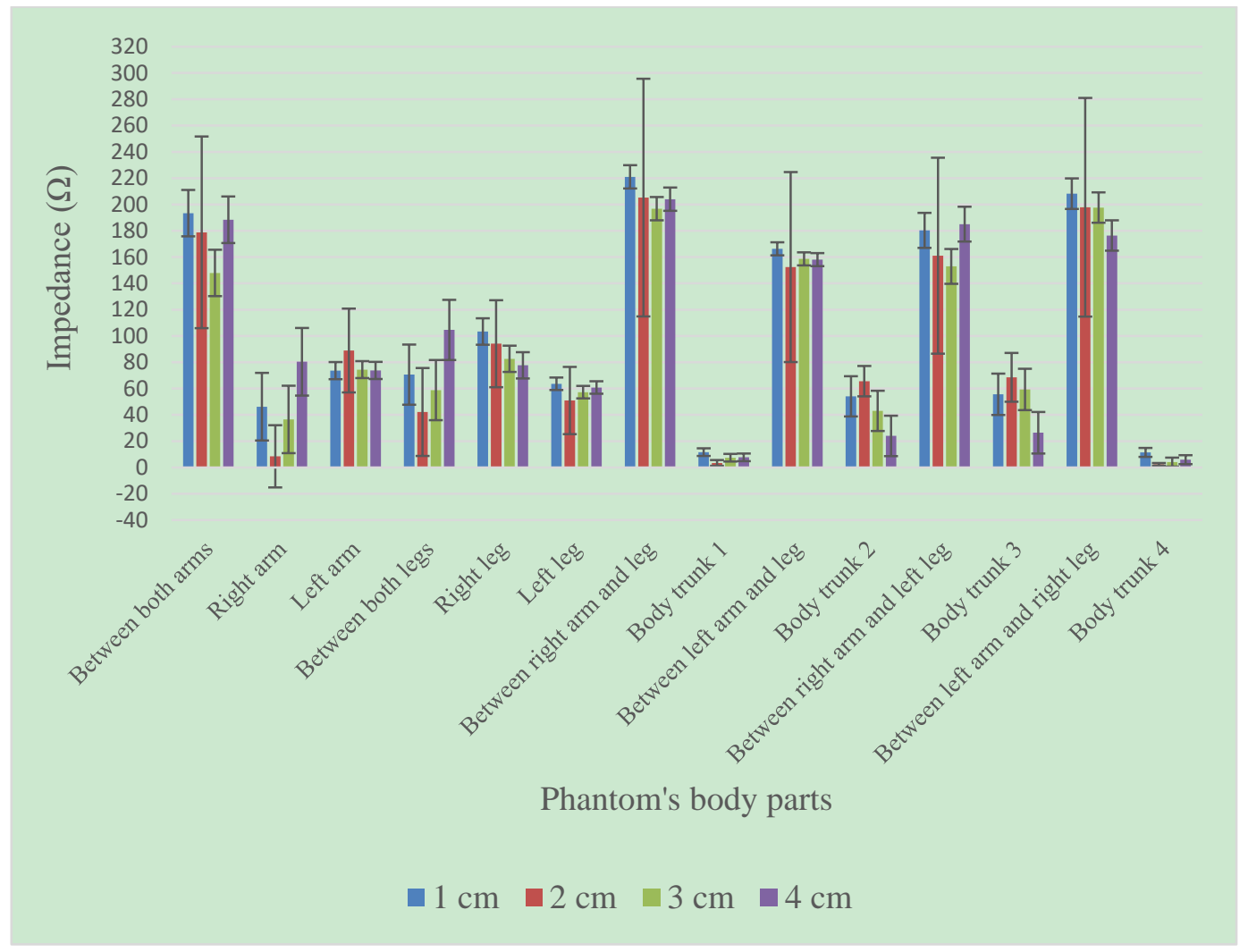

Fig. 4. Impedance at different insertion depths of stainless needle-shaped probes.

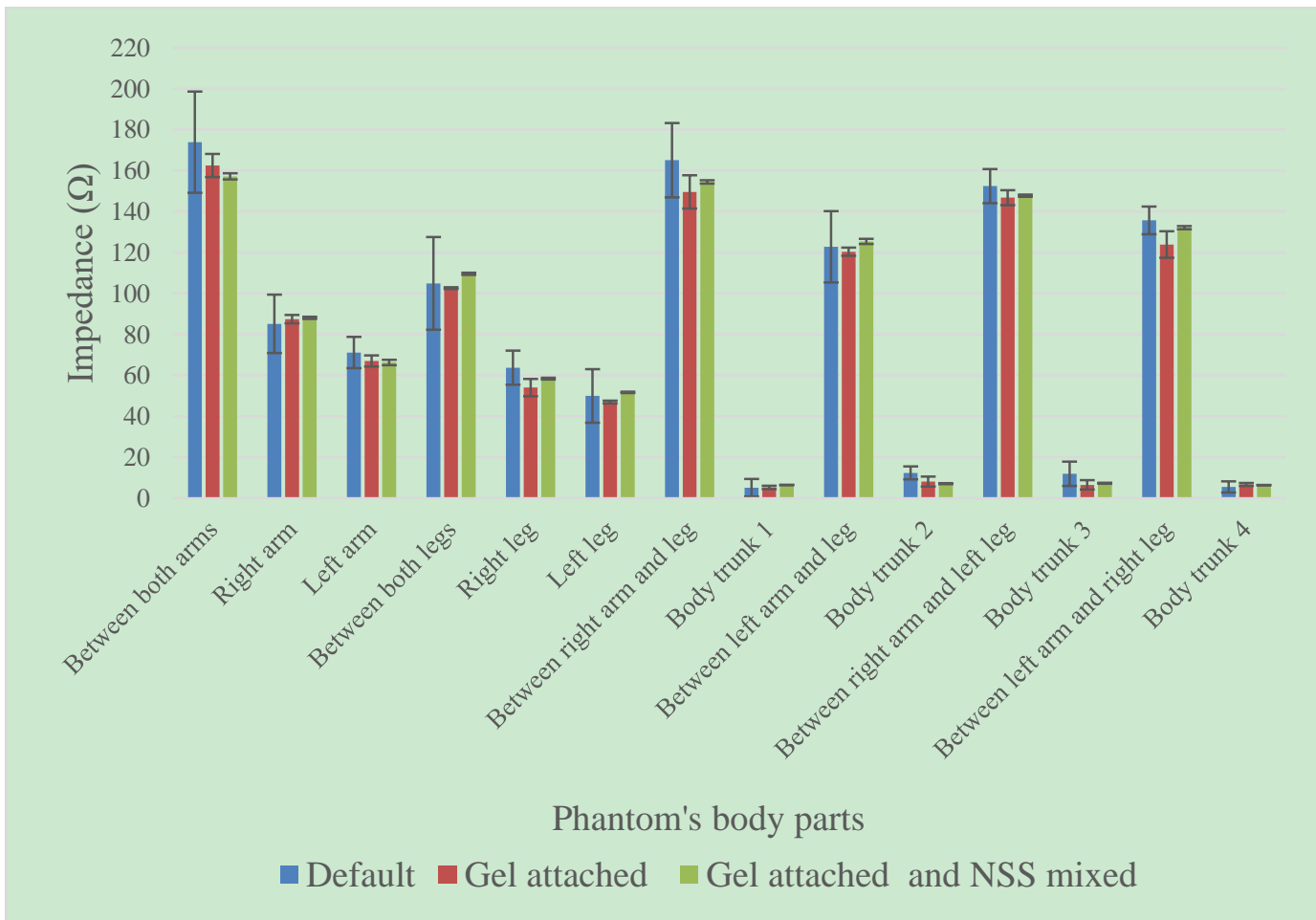

Fig. 5. Impedance under different conditions (default, gel sheet attached, and gel sheet attached with NSS).

In all results, higher impedance values were obtained at the legs than at the arms, which was reasonable 
according to past studies. Previous studies showed that the lowest values were obtained at the upper legs in human limbs, while the upper arms were also rather low-impedance sites; on the other hand, rather high impedance data was measured at the lower legs. In Fig. 4, data obtained from a 1.0 or $4.0 \mathrm{~cm}$ insertion depth of the needle-shaped stainless electrodes were likely to be higher than that of a 2.0 or $3.0 \mathrm{~cm}$ insertion depth. These data mean that the measurement currents selectively run near the surface of the phantom. Fig. 3 and 5 show that the right-hand sides values (i.e., values from the right arm, right leg, and between the right arm and leg,) were slightly higher than left-hand side values. On the other hand, the impedance values of body trunk parts 1 to 4 were similar in Fig. 3 and 5, however, not in Fig. 4. Fig. 5 shows tight data clusters observed between the default, gel attached, and gel attached with NSS conditions. This pattern suggested that these high-frequency impedances $(50 \mathrm{kHz})$ were nearly the same for all electrode locations. Nevertheless, NR gel sheets and NSS could certainly decrease impedance to some extent. Therefore, such chemical substances should be used to make electromagnetically similar dolls or robots.

\section{Discussion and Conclusion}

The authors were able to estimate the impedance of the phantom using the aforementioned methods, and the phantom could be a promising physical simulator with diverse applications at research institutes (e.g., academic sites) and hospitals. During the aforementioned trials, the authors have faced many difficulties; however, their achievements will assist researchers and healthcare workers to overcome those problems more effectively. Additionally, the authors confirmed, reconsidered, and presented various limitations related to performing such measurements. In summary, the authors obtained meaningful results, and presented a phantom with a composition of both superficial and internal isotonic saline (NSS), for which positive benefits were found and reported in the study. The authors wish to confirm the validity, durability, precision, long-term effects, and above all, the social attributes of the system in future work. Additionally, a greater variety of electrical data should be obtained; for example, using phantoms modeled after other races and babies, etc. Furthermore, other methodological variables (e.g., trial timelines, other tools, and specific subjects' conditions, and experimental spaces) should also be investigated in future studies. Regardless of the selection of human models, the diverse problems related to the selection of measuring frequencies will have to be deliberated. The proposed systems have various future prospects, in that it can be used with the latest bioelectronics and bionomics to contribute to improved internal anthropometry. In addition to the phantom's impedance, its hypothetical muscle volumes could also be measured using the aforementioned bioelectrical impedance measurements and analysis. In short, based on data from future studies, relationships could be described between the sizes of each body part, muscle size, and strength in a limited segment of the upper and lower limbs [23], [24], [28]. Furthermore, the launch of practical support for disabled and elderly people must be promising.

\section{Acknowledgment}

Our heartfelt appreciation goes to colleagues at the National Institute of Advanced Industrial Science and Technology (AIST), members of Impulsing Paradigm Change through Disruptive Technologies (ImPACT) Program/Cabinet Office, Government of Japan, for their considerable support, feedback, and valuable comments.

\section{References}

[1] Thomasset, M. A. (1962). Bioelectric properties of tissue. Impedance measurement in clinical medicine. Significance of curves obtained. Lyon medical, 94, 107-118.

[2] Miura, K., Kawagoe, M., \& Koh, S. (2007). Characteristics of functional status among care requiring 
community-dwelling frail elderly. Journal of Human Life Science, 6, 1-10.

[3] Nomoto, K., Miyazaki, R., \& Hasegawa, T. (2010). Efficacy of a health promotion program with anti-aging medical checkup and instructions for walking under pedometer management in factory workers. Anti-Aging Medicine, 7, 73-84.

[4] Suzuki, I. (2011). Study of characteristic and the faculty decline item of the activities of daily living actual situation of nursing care preventive-Service user. Medical and Health Science Research: Bulletin of Tsukuba International University, 2, 167-174.

[5] Miyazaki, R., Hasegawa, T., \& Fujioka, N. (2009). Effects on anti-aging indicators in middle-aged men of an intervention to prevent lifestyle-related diseases: Pilot study utilizing a companywide anti-aging medical checkup and pedometers. Anti-Aging Medicine, 6, 83-94.

[6] Ichikawa, N., \& Tomita, H. (2009). Basic facts about electric shocks and fatal accident statistics for the last 30 years. The National Institute of Occupational Safety and Health, JNIOSH-SD-NO.25.

[7] Nyboer, J. (1959). Electrical impedance plethysmography. Illinois: Charles C Thomas Publisher. Quarterly Journal of Experimental Physiology and Cognate Medical Sciences, 45(3), 322.

[8] Hoffer, E. C., Meador, C. K., \& Simpson, D. C. (1969). Correlation of whole-body impedance with total body water volume. Journal of applied physiology, 27(4), 531-534.

[9] Namie, M., Haeno, M., Sakamoto, K., Kanai, H., \& Tagawa, H. (1985). Estimation of fluid distribution by impedance method. Japanese Society for Medical and Biological Engineering, 23(6), 354-360.

[10] Kim, Y., \& Woo, W. H. (1987). A prototype system and reconstruction algorithms for electrical impedance technique in medical body imaging. Clin. Phys. Physiol. Meas, 8, suppl. A, 63-70.

[11] Colominas, J., Webster, G. J., Rosell, J., Riu, P., \& Pallas-Areny, R. (1988). Skin impedance from $1 \mathrm{~Hz}$ to 1 MHz. IEEE Transactions on Biomedical Engineering, 35(8), 649-651.

[12] Brown, B. H., Karatzas, T., Nakielny, R., \& Clarke, R. G. (1988). Determination of upper arm muscle and fat areas using electrical impedance measurements. Clinical Physics and Physiological Measurement, 9(1), 47-55.

[13] Nakamura, T., Yamamoto, Y., Yamamoto, T., \& Tsuji, H. (1992). Fundamental characteristics of human limb electrical impedance for biodynamic analysis. Medical and Biological Engineering and Computing, 30(5), 465-472.

[14] Foster, K. R., \& Lukaski, H. C. (1996). Whole-body impedance--what does it measure? The American 86 Journal of Clinical Nutrition, 64, 388-396.

[15] Baumgartner, R. N., Ross, R., \& Heymsfield, S. B. (1998). Does adipose tissue influence bioelectric impedance in obese men and women? Journal of applied physiology, 84(1), 257-262.

[16] Baumgartner, R. N., Chumlea, W. C., \& Roche, A. F. (1990). Bioelectric impedance for body composition. Exercise and Sport Sciences Reviews, 18, 193-224.

[17] Abe, T., Kondo, M., Kawakami, Y., \& Fukunaga, T. (1994). Prediction equations for body composition of Japanese adults by B-mode ultrasound. American Journal of Human Biology, 6, 161-170.

[18] Miyatani, M., Kanehisa, H., Masuo, Y., Ito, M., \& Fukunaga, T. (2001). Validity of estimating limb muscle volume by bioelectrical impedance. Journal of Applied Physiology, 91(1), 386-394.

[19] Miyatani, M., Kanehisa, H., Ito, M., Kawakami, Y., \& Fukunaga, T. (2004). The accuracy of volume estimates using ultrasound muscle thickness measurements in different muscle groups. European Journal of Applied Physiology, 91, 264-272.

[20] Gabriel, C., Gabriel, S., \& Corthout, E. (1996). The dielectric properties of biological tissues I. Literature survey. Physics in Medicine and Biology, 41(11), 2231-2249.

[21] Gabriel, C. (1996). The dielectric properties of biological tissue II: Measurements in the frequency range $10 \mathrm{~Hz}$ to $20 \mathrm{GHz}$. Physics in Medicine and Biology, 41(11), 2251-2269. 
[22] Gabriel, C., Gabriel, S., \& Lau, R. (1996). The dielectric properties of biological tissues: III. Parametric models for the dielectric spectrum of tissues. Physics in Medicine and Biology, 41(11), 2271-2293.

[23] Ishiguro, N., Kanehisa, H., Miyatani, M., Masuo, Y., Fukunaga, T. (2005). A comparison of three bioelectrical impedance analyses for predicting lean body mass in a population with a large deference in muscularity. Eur J Appl Physiol, 94, 25-35

[24] Ishiguro, N., Kanehisa, H., Miyatani, M., Masuo, Y., \& Fukunaga, T. (2005). Applicability of segmental bioelectrical impedance analysis for predicting trunk skeletal muscle volume. Journal of Applied Physiology, 100(2), 572-578.

[25] Ministry of Health, Labor and Welfare, Japan. (2013). Public Welfare Statistics Handbook, 2(6).

[26] Gisser, D. G., Isaacson, D., \& Newell, J. C. (1987). Current topics in impedance imaging. Clin. Phys. Physiol. Meas. 8, suppl. A, BME-34, 39-46.

[27] Miyatani, M., Kanehisa, H., \& Fukunaga, T. (2000). Validity of bioelectrical impedance and ultrasonographic methods for estimating the muscle volume of the upper arm. European Journal of Applied Physiology, 82, 391-396.

[28] Tanaka, N. I., Miyatani, M., Masuo, Y., Fukunaga, T., \& Kanehisa, H. (2007). Applicability of a segmental bioelectrical impedance analysis for predicting the whole body skeletal muscle volume. J Appl Physiol, 103, 1688-1695.

[29] Yonei, Y., Miwa, Y., Hibino, S., Takahashi, Y., Miyazaki, R., Yoshikawa, T., Moriwaki, H., Hasegawa, T., Hiraishi, T., \& Torii, K. (2008). Japanese anthropometric reference data special emphasis on bioelectrical impedance analysis of muscle mass. Anti-Aging Medicine, 5(6), 63-72.

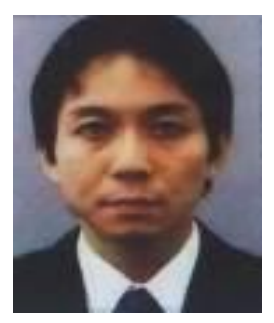

Shinji Kawakura was born on July 14,1978. He is research associate at National Institute of Advanced Industrial Science Technology (AIST), Department of Information Technology and Human Factors. He received the Ph.D. in environmentology, the University of Tokyo, 2015; the B.A in control system engineering, Tokyo Institute of Technology, 2003; the M.A. in human-factors engineering, Tokyo Institute of Technology, 2005. He worked as system engineering, researching for private companies, development and verification of wearable sensing systems with real-time spoken commands for outdoor agricultural workers.

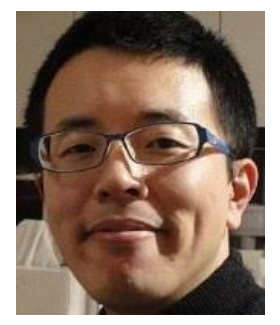

Yoshihiro Nakabo was born on February 22, 1972. He is team leader at National Institute of Advanced Industrial Science and Technology (AIST), Department of Information Technology and Human Factors. He received the Ph.D. in engineering, the University of Tokyo, 2000.

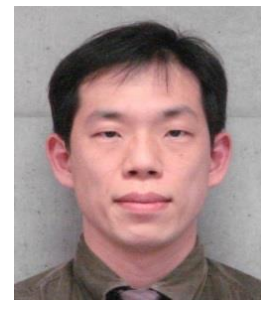

Kiyoshi Fujiwara was born on February 22, 1972. He is senior researcher at National Institute of Advanced Industrial Science and Technology (AIST), Department of Information Technology and Human Factors. He received the Ph.D. in engineering, Tsukuba University. 